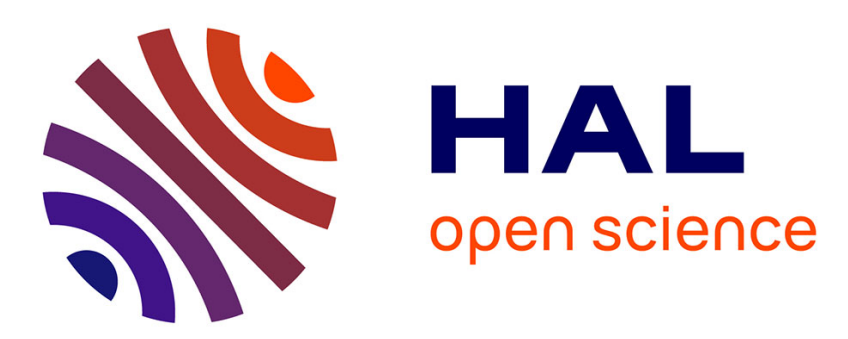

\title{
On the identification of elastoviscoplastic constitutive laws from indentation tests
}

Andreï Constantinescu, Nicolas Tardieu

\section{To cite this version:}

Andreï Constantinescu, Nicolas Tardieu. On the identification of elastoviscoplastic constitutive laws from indentation tests. Inverse Problems in Engineering, 2001, 9, pp.19-44. 10.1080/174159701088027751 . hal-00111325

\section{HAL Id: hal-00111325 \\ https://hal.science/hal-00111325}

Submitted on 15 Aug 2019

HAL is a multi-disciplinary open access archive for the deposit and dissemination of scientific research documents, whether they are published or not. The documents may come from teaching and research institutions in France or abroad, or from public or private research centers.
L'archive ouverte pluridisciplinaire HAL, est destinée au dépôt et à la diffusion de documents scientifiques de niveau recherche, publiés ou non, émanant des établissements d'enseignement et de recherche français ou étrangers, des laboratoires publics ou privés.

\section{다(1) $\$$}

Distributed under a Creative Commons Attribution - NonCommercial| 4.0 International 


\title{
ON THE IDENTIFICATION OF ELASTOVISCOPLASTIC CONSTITUTIVE LAWS FROM INDENTATION TESTS
}

\author{
ANDREI CONSTANTINESCU* and NICOLAS TARDIEU \\ Laboratoire de Mécanique des Solides, CNRS UMR 7649, Ecole Polytechnique, \\ 91128 Palaiseau Cedex, France
}

\begin{abstract}
This paper addresses the identification of the parameters of a nonlinear constitutive law from indentation tests. The identification problem is considered as a constrained minimization problem and the gradient is computed using the adjoint state method, in spite of the difficulties of the underlying contact problem. This provides a general framework to perform optimization in some problems involving contact conditions and nonlinear material behaviour. The case of a Maxwell viscoelastic and a Norton-Hoff elastoviscoplastic constitutive law are treated extensively and a series of numerical identification examples are shown.
\end{abstract}

Keywords:

\section{INTRODUCTION}

The indentation test is performed by pressing a punch into a material sample. It was initialy used to evaluate the hardness of metals and is now being considered as an efficient non-destructive method for determining mechanical characteristics of materials [19].

The constitutive law should be identified from the knowledge of the indentation curve (see Fig. 3), which represents the load applied on the punch versus the penetration depth. The applicability of the

\footnotetext{
*Corresponding author. e-mail: constant; tardieu@1ms. polytechnique.fr
} 
indentation tests depends at this point on the precision and generality of this identification.

The identification strategies currently used in the experiments are based on semi-empirical formulas dedicated to given constitutive behaviour: elasticity, perfect plasticity [12], power laws [11],... These formulas are based on elastoplastic flow theories as presented in the classical monograph of Tabor [18].

Only a few studies present a general method applicable to a large class of constitutive behaviours, like defining the identification as the minimization of a cost functional.

Koguchi [14] identified the elastic coefficients of a layered half-space indented with a sphere, minimizing a least squares distance between measurements and computations using a closed form solution to the direct problem.

More generally, for a non-linear elastic constitutive law and a least squares cost functional Hasanov [7-9] proved some existence results and proposed a numerical procedure based on trial and error.

The previous methods are dedicated essentially to some elastoplastic behaviours and the minimizations do not include gradient computations of the cost functional. Gradient computations are difficult due to the mathematical complexity of the contact description [10,13], appearing independently of the constitutive behaviour of the material. As the direct problem is numerically costly, the knowledge of the gradient could speed up the minimization algorithm.

The computation of a gradient of a least squares functional in the case of linear elasticity has been given in $[4,20]$. It has been shown that the adjoint state method [15] can be extended to variational inequalities. The efficiency of the method has been illustrated by numerical examples in the identification of linear elastic moduli from indentation tests.

The goal of this paper is to show that the gradient computation by an adjoint state method can be used in combination with a nonlinear elastoviscoplastic constitutive law under the assumption of small strains and rotations. Moreover, the method applies to any sufficiently regular standard generalized constitutive law (for a presentation of these behaviours, see $[1,6])$. This ensures the generality of the approach.

As a consequence the parameters of nonlinear elastoviscoplastic constitutive laws can be identified from indentation tests by minimizing least squares functionals with gradient algorithms. 
The accuracy and robustness of the method is illustrated in this paper through numerical examples for a Maxwell viscoelastic and a Norton-Hoff viscoplastic constitutive law.

\section{THE DIRECT PROBLEM $(\mathcal{P})$}

Let us consider an axisymmetric body, with its section occupying in its reference configuration an open subset $\Omega \subset \mathbb{R}^{2}$ with smooth boundary $\Gamma$ (see Fig. 1). The boundary is partioned in three parts $\Gamma=\Gamma_{D} \cup \Gamma_{F} \cup \Gamma_{C}$ : the part $\Gamma_{\mathrm{D}}$ where displacements are imposed, the free surface $\Gamma_{F}$, and the surface $\Gamma_{C}$ where contact might occur. $\mathbf{n}$ and $\mathbf{t}$ denote the normal and tangent vector to the boundary $\Gamma$.

The axisymmetric hypothesis is taken in order to simplify the presentation and the computational burden and does not restrict the generality of the method.

The problem will be treated within the theory of small strains and rotations. The validity of this hypothesis will be discussed later. Therefore, let us denote respectively by $\mathbf{u}, \varepsilon$ and $\boldsymbol{\sigma}$ the vector field of displacements and the tensor fields of small strains and stresses.

The problem considered in the sequel is the indentation of the body $\Omega$ by a rigid punch whose profile is characterized by the gap $g$. For a

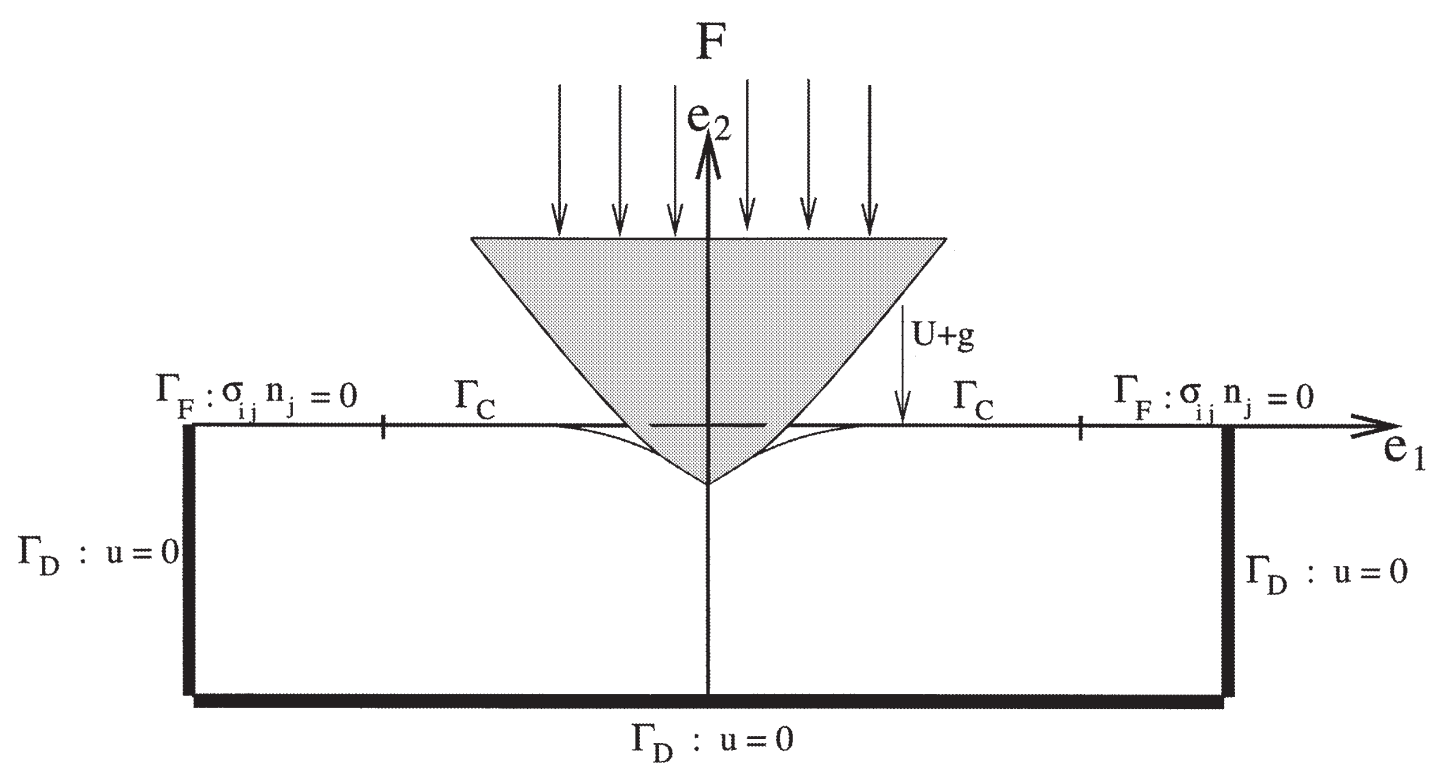

FIGURE 1 The configuration of the direct problem for a conical punch. 
complete presentation of contact computations see [13]. The contact is considered without friction.

An indentation experiment is driven either by the vertical displacement $U$ or by the force $F$ applied to the punch. The force $F$ can be expressed as integral of the contact pressure:

$$
F=\int_{\Gamma_{C}} \mathbf{n} \cdot \boldsymbol{\sigma} \cdot \mathbf{n} d \Gamma
$$

An experiment provides an indentation curve (see Fig. 3), representing a displacement-force history $\left(U^{\exp }, F^{\text {exp }}\right)$ over a given time interval $[0, T]$.

In this work, we have always expressed the problem as driven by the punch displacement. The governing equations can be written using:

- a time continous expression, where the intervening quantities are the velocities of the fields, or

- a time discretized expression, where the intervening quantities are small increments of the fields between two time steps.

Describing contact conditions using velocities of the fields is a complicated task demanding care in the choice of the functional spaces of the mathematical formulations [13]. Therefore the time discretized expression, which permits to avoid some of these difficulties, will be used in this paper.

\subsection{Constitutive Law}

A standard generalized material without work hardening [6] is considered here. This constitutive behaviour is completely determined by the elasticity tensor $\mathbf{S}(\mathbf{c})$ and by the pseudo-potential of dissipation $\Phi=\Phi(\boldsymbol{\sigma}, \mathbf{c})$. The later is supposed to be twice differentiable with respect to $\boldsymbol{\sigma} . \mathbf{c}$ is the vector of the material parameters characterizing the material behaviour (Young's modulus, elasticity limit, ...).

\subsubsection{Time Continuous Expression}

In a time continuous description, the constitutive law is expressed by the classical set of equations: 


$$
\begin{gathered}
\varepsilon(\dot{\mathbf{u}})=\mathbf{S}(\mathbf{c}): \dot{\boldsymbol{\sigma}}+\dot{\varepsilon}^{p} \\
\dot{\varepsilon}^{p}=\frac{\partial \Phi(\boldsymbol{\sigma}, \mathbf{c})}{\partial \boldsymbol{\sigma}}
\end{gathered}
$$

where the dot denotes the time derivative and $\varepsilon^{p}$ is the viscoplastic strain.

\subsubsection{Time Discretized Expression}

In a time discretization, the previous equations are expressed as

$$
\begin{aligned}
\varepsilon\left(\Delta \mathbf{u}_{i}\right) & =\mathbf{S}: \Delta \boldsymbol{\sigma}_{i}+\Delta \varepsilon_{i}^{p} \\
\Delta \varepsilon_{i}^{p} & =\frac{\partial \Phi\left(\boldsymbol{\sigma}_{i}, \mathbf{c}\right)}{\partial \boldsymbol{\sigma}} \Delta t
\end{aligned}
$$

Examples The following classical constitutive laws can be expressed under this formalism:

- The Maxwell viscoelastic material : the pseudo potential $\Phi$ is given by $\Phi\left(\boldsymbol{\sigma}_{i}, \mathbf{c}\right)=(1 / 2) \boldsymbol{\sigma}_{i}: \mathbf{M}: \boldsymbol{\sigma}_{i}$ where $\mathbf{M}$ is a fourth order tensor. The plastic strain increment is determined by : $\Delta \varepsilon_{i}^{p}=\mathbf{M}: \boldsymbol{\sigma}_{i} \Delta t$.

- The Norton-Hoff viscoplastic material: the pseudo potential $\Phi$ is given by $\left.\Phi\left(\boldsymbol{\sigma}_{i}, \mathbf{c}\right)=(K /(m+1))\left\langle\left(\left(\boldsymbol{\sigma}_{i}\right)_{\mathrm{eq}}-\sigma^{Y}\right) / K\right)\right\rangle_{+}^{m+1}$ where $\sigma^{Y}$ is the elasticity limit, $\langle\cdot\rangle_{+}$is the positive part operator and $(\cdot)_{\mathrm{eq}}$ is the equivalent Mises stress. The plastic strain increment is determined by: $\Delta \varepsilon_{i}^{p}=(3 / 2)\left\langle\left(\left(\sigma_{i}\right)_{\mathrm{eq}}-\sigma^{Y}\right) / K\right\rangle_{+}^{m}\left(\tilde{\boldsymbol{\sigma}}_{i} /\left(\sigma_{i}\right)_{\mathrm{eq}}\right) \Delta t ; \tilde{\boldsymbol{\sigma}}_{i}$ is the deviator of $\boldsymbol{\sigma}_{i}$.

\subsection{Equations of the Direct Problem $(\mathcal{P})$}

The governing equations of the direct problem consist of the equilibrium and constitutive equations, the boundary and contact conditions and a set of initial values. The contact conditions on $\Gamma_{C}$ are expressed using the Lagrange multipliers $p_{i} \in N$, where $N=\left\{q \in\left(H^{1 /}\right.\right.$ $\left.\left.{ }^{2}\left(\Gamma_{C}\right)\right)^{\prime} \mid q \leq 0\right\}$ is a closed convex set and $\left(H^{1 / 2}\left(\Gamma_{C}\right)\right)^{\prime}$ denotes the dual of $H^{1 / 2}\left(\Gamma_{C}\right)$ (see [13]).

The punch is driven by its vertical displacement $U_{i}$ at time $t_{i}$. At the same moment, the gap $g_{i}$ between the surface $\Gamma_{C}$ and the punch is expressed by $g_{i}=g+U_{i}-u_{i}^{n}$. 
Equilibrium and Constitutive Equation in $\mathbf{\Omega}$

$$
\begin{gathered}
\operatorname{div}\left(\Delta \boldsymbol{\sigma}_{i}\right)=0 \\
\varepsilon\left(\Delta \mathbf{u}_{i}\right)=\mathbf{S}: \Delta \boldsymbol{\sigma}_{i}+\frac{\partial \Phi\left(\boldsymbol{\sigma}_{i}, \mathbf{c}\right)}{\partial \boldsymbol{\sigma}} \Delta t
\end{gathered}
$$

Boundary Conditions

$$
\begin{gathered}
\Delta \boldsymbol{\sigma}_{i} \cdot \mathbf{n}=0 \text { on } \Gamma_{F} \\
\Delta \mathbf{u}_{i}=0 \text { on } \Gamma_{D}
\end{gathered}
$$

Contact Conditions

$$
\begin{gathered}
\left(\Delta u_{i}^{n}-g_{i}-\Delta U_{i}\right)\left(q-p^{i+1}\right) \geq 0 \quad \forall q \in N \\
\Delta \sigma_{i}^{n n}=\Delta \boldsymbol{\sigma}_{i} \cdot \mathbf{n} \cdot \mathbf{n}=\Delta p_{i} \\
\Delta \sigma_{i}^{n t}=\left(\Delta \boldsymbol{\sigma}_{i} \cdot \mathbf{n}-\Delta \sigma_{i}^{n n} \cdot \mathbf{n} \cdot \mathbf{t}=0\right.
\end{gathered}
$$

Initial Conditions

$$
\begin{gathered}
\boldsymbol{\sigma}_{0}=0 \text { in } \Omega \\
\mathbf{u}_{0}=0 \text { on } \Omega \\
\varepsilon_{0}^{p} \cdot \mathbf{n}=0 \text { on } \Omega
\end{gathered}
$$

\section{THE INVERSE PROBLEM $\left(\mathcal{P}^{-1}\right)$}

In the present inverse problem, one wants to identify the parameters of the material behaviour $\mathbf{c}$ from the knowledge of the indentation curve $\left(U^{\exp }, F^{\exp }\right)$. $\mathbf{c}$ is supposed to evaluate in a closed convex $\mathcal{Q}$ of $\mathbb{R}^{n}$ $(n \geq 2)$.

This inverse problem can be expressed as a minimization problem of a well chosen cost functional. Since the direct problem is driven by the 
imposed displacement of the punch $U$, it is natural to express the cost functional as a function of the resultant force $F$. A possible formulation of the inverse problem $\left(\mathcal{P}^{-1}\right)$ is:

Find $c \in \mathcal{Q}$ minimizing

$$
\begin{aligned}
\mathcal{J}(\mathbf{c}) & =\frac{1}{2} \sum_{i=0}^{I}\left(F_{i}^{\mathrm{comp}}(\mathbf{c})-F_{i}^{\exp }\right)^{2} \\
& =\frac{1}{2} \sum_{i=0}^{I}\left(\int_{\Gamma_{C}} p_{i}(\mathbf{c}) d \Gamma-F_{i}^{\exp }\right)^{2}
\end{aligned}
$$

where, $F^{\text {comp }}$ is the computed resultant force from the direct problem driven by $U^{\exp }$.

One can remark that the cost functional $\mathcal{J}$ depends implicitly on the material parameters $\mathbf{c}$ through the intermediate of the pressure distribution $p$. The resolution of the direct problem $(\mathcal{P})$ permits the determination of the Lagrange multiplier $p_{i}$ and then the calculation of $F_{i}^{\text {calc }}$. In consequence, this minimization problem can be considered as a constrained one, the constraint being the resolution of $(\mathcal{P})$.

In the case of an elastoplastic constitutive law existence results for this problem are given in [7-9].

\subsection{Resolution}

The resolution of a constrained minimization problem is equivalent, under some regularity conditions, to finding the saddle point of a Lagrangian functional $\mathcal{L}$. In the case of the inverse problem $\left(\mathcal{P}^{-1}\right)$, the Lagrangian $\mathcal{L}$ is introduced as a sum between the cost functional and a variational formulation of the direct problem $(\mathcal{P})$.

For each variable of the direct problem, an adjoint variable, denoted by $\mathrm{a} \star$ superscript, is introduced. These adjoint variables are the Lagrange multipliers of the constraints, the equations of the direct problem.

According to the optimal control theory, all direct and adjoint variables will be considered mutually independent. The relationships between them will be recovered from the stationarity conditions of the Lagrangian $\mathcal{L}$, characterizing the saddle point.

The Lagrangian functional has the following form: 


$$
\mathcal{L}\left(\mathbf{u}, \boldsymbol{\sigma}, p, \mathbf{u}^{\star}, \boldsymbol{\sigma}^{\star}, p^{\star}, \mathbf{c}\right)=\sum_{i=0}^{I} \mathcal{L}_{i}\left(\mathbf{u}_{i}, \boldsymbol{\sigma}_{i}, p_{i}, \mathbf{u}_{i}^{\star}, \boldsymbol{\sigma}_{i}^{\star}, p_{i}^{\star}, \mathbf{c}\right)
$$

where

$$
\begin{aligned}
& \mathcal{L}_{i}\left(\mathbf{u}_{i}, \boldsymbol{\sigma}_{i}, p_{i}, \mathbf{u}_{i}^{\star}, \boldsymbol{\sigma}_{i}^{\star}, p_{i}^{\star}\right) \\
= & \frac{1}{2}\left(\int_{\Gamma_{C}} p_{i} d \Gamma-F_{i}^{\exp }\right)^{2} \int_{\Omega} \operatorname{div}\left(\Delta \boldsymbol{\sigma}_{i}\right) \cdot \mathbf{u}_{i}^{\star} d \Omega \\
& \int_{\Gamma_{F}} \Delta \boldsymbol{\sigma}_{i} \cdot \mathbf{n} \cdot \mathbf{u}_{i}^{\star} d \Gamma+\int_{\Gamma_{D}} \Delta \mathbf{u}_{i} \cdot \boldsymbol{\sigma}_{i}^{\star} \cdot \mathbf{n} d \Gamma \\
& \left.\int_{\Gamma_{C}}\left(\Delta u_{i}^{n}-\Delta U_{i}-g\right)_{i}\right) \cdot p_{i}^{\star} d \Gamma-\int_{\Omega}\left(\varepsilon\left(\Delta \mathbf{u}_{i}\right)-\mathbf{S}: \Delta \boldsymbol{\sigma}_{i}\right. \\
& \left.\frac{\partial \Phi\left(\boldsymbol{\sigma}_{i}, \mathbf{c}\right)}{\partial \boldsymbol{\sigma}} \Delta t\right): \boldsymbol{\sigma}_{i}^{\star} d \Omega \\
& \int_{\Gamma_{C}}\left(\Delta p_{i}-\Delta \boldsymbol{\sigma}_{i}^{n n}\right) \cdot u_{i}^{n \star} d \Gamma \\
& \int_{\Gamma_{C}} \Delta \sigma_{i}^{n t} \cdot u_{i}^{t \star} d \Gamma
\end{aligned}
$$

and

- $\mathbf{u}_{i}, \mathbf{u}_{i}^{\star} \in\left(H^{1}(\Omega)\right)^{2}$

- $\boldsymbol{\sigma}_{i}, \boldsymbol{\sigma}_{i}^{\star} \in\left(L^{2}(\Omega)\right)^{4}$

- $p_{i}, p_{i}^{\star} \in N_{i}=\left\{q \in\left(H^{1 / 2}\right)^{\prime}\left(\Gamma_{C}\right) \mid q=0\right.$ on $\left.\Gamma_{C} / \Gamma_{C_{i}}\right\}$ and where $\Gamma_{C_{i}}$ is the effective contact surface at time $t_{i}$.

The complex form of this Lagrangian does not permit to draw any conclusions with regard to the existence and uniqueness of its saddle point. Nevertheless, necessary conditions of stationarity can be formally written in order to characterize this eventual saddle point.

The stationarity conditions of $\mathcal{L}$ are given by the following expressions:

$$
\begin{array}{ll}
\sum_{i=0}^{I}\left\langle\frac{\partial \mathcal{L}_{i}}{\partial \mathbf{u}}, d \mathbf{w}_{i}\right\rangle=0 & \forall d \mathbf{w}_{i} \in\left(H^{1}(\Omega)\right)^{2} \\
\sum_{i=0}^{I}\left\langle\frac{\partial \mathcal{L}_{i}}{\partial \boldsymbol{\sigma}}, d \boldsymbol{\tau}_{i}\right\rangle=0 & \forall d \boldsymbol{\tau}_{i} \in\left(L^{2}(\Omega)\right)^{4}
\end{array}
$$




$$
\begin{gathered}
\sum_{i=0}^{I}\left\langle\frac{\partial \mathcal{L}_{i}}{\partial p}, d q_{i}\right\rangle=0 \quad \forall d q_{i} \in N_{i} \\
\sum_{i=0}^{I}\left\langle\frac{\partial \mathcal{L}_{i}}{\partial \mathbf{u}^{\star}}, d \mathbf{w}_{i}\right\rangle=0 \quad \forall d \mathbf{w}_{i} \in\left(H^{1}(\Omega)\right)^{2} \\
\sum_{i=0}^{I}\left\langle\frac{\partial \mathcal{L}_{i}}{\partial \boldsymbol{\sigma}^{\star}}, d \boldsymbol{\tau}_{i}\right\rangle=0 \quad \forall d \boldsymbol{\tau}_{i} \in\left(L^{2}(\Omega)\right)^{4} \\
\sum_{i=0}^{I}\left\langle\frac{\partial \mathcal{L}_{i}}{\partial p^{\star}}, d q_{i}\right\rangle=0 \quad \forall d q_{i} \in N_{i} \\
\sum_{i=0}^{I}\left\langle\frac{\partial \mathcal{L}_{i}}{\partial \mathbf{c}}, \mathbf{d}-\mathbf{c}\right\rangle \geq 0 \quad \forall \mathbf{d} \in \mathcal{Q}
\end{gathered}
$$

where $\langle\cdot, \cdot\rangle$ represents in each equation the duality pairing for the corresponding functional spaces.

Calculating the derivatives with respect to the adjoint variables (Eqs. (21)-(23)) leads to the set of equations:

$$
\begin{gathered}
\operatorname{div}\left(\Delta \boldsymbol{\sigma}_{i}\right)=0 \text { in } \Omega \\
\varepsilon\left(\Delta \mathbf{u}_{i}\right)-\mathbf{S}: \Delta \boldsymbol{\sigma}_{i}-\frac{\partial \Phi\left(\boldsymbol{\sigma}_{i}, \mathbf{c}\right)}{\partial \boldsymbol{\sigma}} \Delta t=0 \text { in } \Omega \\
\Delta \boldsymbol{\sigma}_{i} \cdot \mathbf{n}=0 \text { on } \Gamma_{F} \\
\Delta \mathbf{u}_{i}=0 \text { on } \Gamma_{D} \\
\left.\begin{array}{c}
\Delta u_{i}^{n}-g_{i}-\Delta U_{i}=0 \\
\Delta \boldsymbol{\sigma}_{i}^{n n}=\Delta p_{i} \\
\Delta \sigma_{i}^{n t}=0
\end{array}\right\} \text { on } \Gamma_{C_{i}}
\end{gathered}
$$

The preceding calculation leads in classical Lagrangian theory to the equations of the direct problem. In the present case, opposite to the classical frame, the equations do not represent exactly the direct problem. However, if $(\mathbf{u}, \boldsymbol{\sigma}, p)$ are the solutions to $(\mathcal{P})$, they obviously verify the above relations. 
The derivation of $\mathcal{L}$ with respect to the direct variables (Eqs. (18)(20)) and series of calculations: spatial integration by parts and use of the first order approximation $f_{i} \cdot \Delta g_{i} \approx f^{i+1} \cdot g^{i+1}-f^{i} \cdot g^{i}-\Delta f_{i} \cdot g^{i}$, gives the following set of equations:

$$
\begin{gathered}
\operatorname{div}\left(\Delta \boldsymbol{\sigma}_{i}\right)=0 \text { in } \Omega \\
\varepsilon\left(\Delta \mathbf{u}_{i}^{\star}\right)=\mathbf{S}: \Delta \boldsymbol{\sigma}_{i}^{\star}-\frac{\partial^{2} \Phi\left(\boldsymbol{\sigma}_{i}, \mathbf{c}\right)}{\partial \boldsymbol{\sigma}^{2}} \Delta t: \boldsymbol{\sigma}_{i}^{\star} \text { in } \Omega \\
\Delta \boldsymbol{\sigma}_{i}^{\star} \cdot \mathbf{n}=0 \text { on } \Gamma_{F} \\
\Delta \mathbf{u}_{i}^{\star}=0 \text { on } \Gamma_{D} \\
\left.\begin{array}{c}
\Delta u_{i}^{n \star}=\left(F_{i}^{\text {calc }}(\mathbf{c})-F_{i}^{\exp }\right) \\
\Delta \sigma_{i}^{n \hbar \star}=\Delta p_{i}^{\star} \\
\Delta \sigma_{i}^{n \star \star}=0
\end{array}\right\} \text { on } \Gamma_{C_{i}}
\end{gathered}
$$

and the following final conditions at time $T$

$$
\begin{aligned}
& \operatorname{div}\left(\boldsymbol{\sigma}_{I}^{\star}\right)=0 \text { in } \Omega \\
& \varepsilon\left(\Delta \mathbf{u}_{I}^{\star}\right)=\mathbf{S}: \Delta\left(\boldsymbol{\sigma}_{I}^{\star}\right) \text { in } \Omega \\
& \mathbf{u}_{I}^{\star}=0 \text { on } \Gamma_{D} \\
& \boldsymbol{\sigma}_{I}^{\star} \cdot \mathbf{n}=0 \text { on } \Gamma_{F} \\
& \left.\begin{array}{l}
u_{i}^{n \star}=0 \\
p_{I}^{\star}=\sigma_{I}^{n n \star}
\end{array}\right\} \text { on } \Gamma_{C}
\end{aligned}
$$

This set of equations and the final conditions define a well-posed incremental problem with Dirichlet conditions on a part of the boundary and will be called the adjoint problem $\left(\mathcal{P}^{\star}\right)$.

The preceding calculations can be summarized as:

\subsection{Stationarity Result}

If $(\mathbf{u}, \boldsymbol{\sigma}, p)$ and $\left(\mathbf{u}^{\star}, \boldsymbol{\sigma}^{\star}, p^{\star}\right)$ are respectively the solutions to the incremental direct and adjoint problem $(\mathcal{P})$ and $\left(\mathcal{P}^{\star}\right)$, then the Conditions (18) $-(23)$ of stationarity of the Lagrangian $\mathcal{L}$ are verified. 
Moreover, if $(\mathbf{u}, \boldsymbol{\sigma}, p)$ are the solutions to $(\mathcal{P})$, one can notice that the Lagrangian $\mathcal{L}$ is reduced to the cost functional $\mathcal{J}$. Together with the expression of stationarity conditions (24) this implies that:

\subsection{Gradient Computation}

If $(\mathbf{u}, \boldsymbol{\sigma}, p)$ and $\left(\mathbf{u}^{\star}, \boldsymbol{\sigma}^{\star}, p^{\star}\right)$ are respectively the solutions to the incremental direct problem $(\mathcal{P})$ and to the incremental adjoint problem $\left(\mathcal{P}^{*}\right)$, then the gradient of the cost functional $\mathcal{J}$ is given by

$$
\nabla_{c} \mathcal{J}=\sum_{i=0}^{I}\left(\int_{\Omega} \Delta \boldsymbol{\sigma}_{i}: \frac{\partial \mathbf{S}}{\partial \mathbf{c}}: \boldsymbol{\sigma}_{i}^{\star}+\frac{\partial^{2} \Phi}{\partial \boldsymbol{\sigma} \partial \mathbf{c}} \Delta t: \boldsymbol{\sigma}_{i}^{\star} d \Omega\right)
$$

Some remarks can be done about the preceeding results:

- The adjoint problem is not a contact problem. Its loading is a Dirichlet conditions (imposed displacement) on $\Gamma_{C_{i}}$, the effective contact surface of the direct problem.

- The adjoint problem is a time dependent system of partial differential equations on $[0, T]$ and a final condition given by a well-posed elasticity problem. Therefore the adjoint problem will be integrated from $T$ to 0 in the reversed time.

- The adjoint constitutive law is viscoelastic considered in the reversed time $i^{\prime} \leftarrow I-i$ :

$$
\varepsilon\left(\Delta \mathbf{u}_{i^{\prime}}^{\star}\right)=\mathbf{S}: \Delta \boldsymbol{\sigma}_{i^{\prime}}^{\star}+\mathbf{R}: \boldsymbol{\sigma}_{i^{\prime}}^{\star} \Delta t
$$

where $\mathbf{R}$ is the forth order tensor:

$$
\mathbf{R}=\frac{\partial^{2} \Phi\left(\boldsymbol{\sigma}_{i^{\prime}}, \mathbf{c}\right)}{\partial \boldsymbol{\sigma}_{i^{\prime}}^{2}}
$$

The pseudo potential $\Phi$ has to be twice differentiable to ensure the existence of the adjoint state (For example in the Norton-Hoff constitutive law, $m>1$ is needed). From a numerical point of view, this leads to a linear problem at each time step and therefore a rapid integration.

- The parameters to the adjoint constitutive law depend on the parameters of the direct constitutive law, but also on the solution of 
the direct problem. Hence the solution to the adjoint problem is implicitly dependent on the solution of the direct problem.

- The equations of the adjoint problem in the reversed time describe a linear viscoelastic problem with Dirichlet boundary conditions and initial conditions, and therefore well posed.

- This method allows the computation of the gradient of the cost functional $\mathcal{J}$ using the solutions to the direct and adjoint problems, independently of the number of parameters involved. A rapid evaluation of the computational burden shows that a gradient calculation takes $\approx 1.5$ the time for solving the direct problem due to the simplicity of the adjoint behaviour and the elimination of the contact condition. This is extremely interesting for problems with a large number of parameters. However, the intervention of the solution to the direct problem in the resolution of the adjoint problem, demands large memory space for keeping track of all the fields.

\section{NUMERICAL EXAMPLE FOR THE MAXWELL EQUATION}

In order to illustrate the presented method, let us consider the identification of the parameters of a Maxwell viscoelastic material:

$$
\begin{gathered}
\varepsilon\left(\Delta \mathbf{u}_{i}\right)=\mathbf{S}: \Delta \boldsymbol{\sigma}_{i}+\Delta \varepsilon_{i}^{p} \quad \text { where } \Delta \varepsilon_{i}^{p}=\mathbf{M}: \boldsymbol{\sigma}_{i} \Delta t \\
S_{i j k l}=\frac{1}{E}\left((1+\nu) \delta_{i k} \delta_{j l}-\nu \delta_{k l} \delta_{i j}\right) \\
M_{i j k l}=\frac{1}{\eta}\left(\delta_{i k} \delta_{j l}-\frac{1}{3} \delta_{k l} \delta_{i j}\right)
\end{gathered}
$$

where $E, \nu, \eta$ denote respectively the Young's modulus, the Poisson coefficient and the viscosity. The tensor $\mathbf{M}$ computes the deviatoric part of stress.

As explained before, this constitutive law enters the formalism of the standard generalized materials without work-hardening.

The identification problem consists of determining $E$ and $\eta$ from an indentation curve ( $U^{\exp }, F^{\exp }$ ). The Poisson coefficient was considered as known, but could be identified in the same way. 
In this work the indentation curve is obtained from numerical experiments as explained in the sequel.

\subsection{Direct Calculations}

The experiment simulations have been realized through finite elements computations using the CASTEM2000 code [3]. The body $\Omega$ was a cylinder with radius $10 \mathrm{~mm}$ and height $10 \mathrm{~mm}$ and the punch was a rigid cone with a $68^{\circ}$ half angle at the apex. The mesh of the body had $20 \times 20$ quadratic elements. At this coarness the solution of the direct problem is independent of the mesh size.

The indentation process has been displacement controlled and consists of a loading, maintain and unloading parts (see Fig. 2). A typical indentation curve is represented on Figure 3.

The hypothesis of small strains and rotations has been the keypoint in the development of the calculations of the adjoint state method. It was therefore important to validate this assumption. A series of direct computations have been done in three different cases: small strain and rotations, large strains and large strains and rotations, with $E=2$ $10^{4} \mathrm{MPa}$ and $\eta=310^{4} \mathrm{MPa} \cdot \mathrm{s}$. The results show a good agreement of the indentation curves (see Fig. 3). It is important to remark that the small difference is due in part to the simple constitutive law assumed. This hypothesis should be checked before applying this method for other constitutive laws.

\subsection{Identification Procedure}

The identification procedure presented next is based on minimization of the cost functional $\mathcal{J}$ (16) using a gradient descent method. The "experimental" curve was simulated by finite element calculations as stated in the previous section with $E=210^{4} \mathrm{MPa}, \nu=0.3, \eta=3$ $10^{4} \mathrm{MPa} \cdot \mathrm{s}$.

The gradient has been computed using the adjoint state method with the expression (40) after solving the direct problem $(\mathcal{P})$ and the adjoint problem $\left(\mathcal{P}^{\star}\right)$. In the case of the Maxwell material behaviour the adjoint behaviour is also a Maxwell law, which we might call a self adjoint material behaviour. This is due to the quadratic viscoelastic potential $\Phi$. 


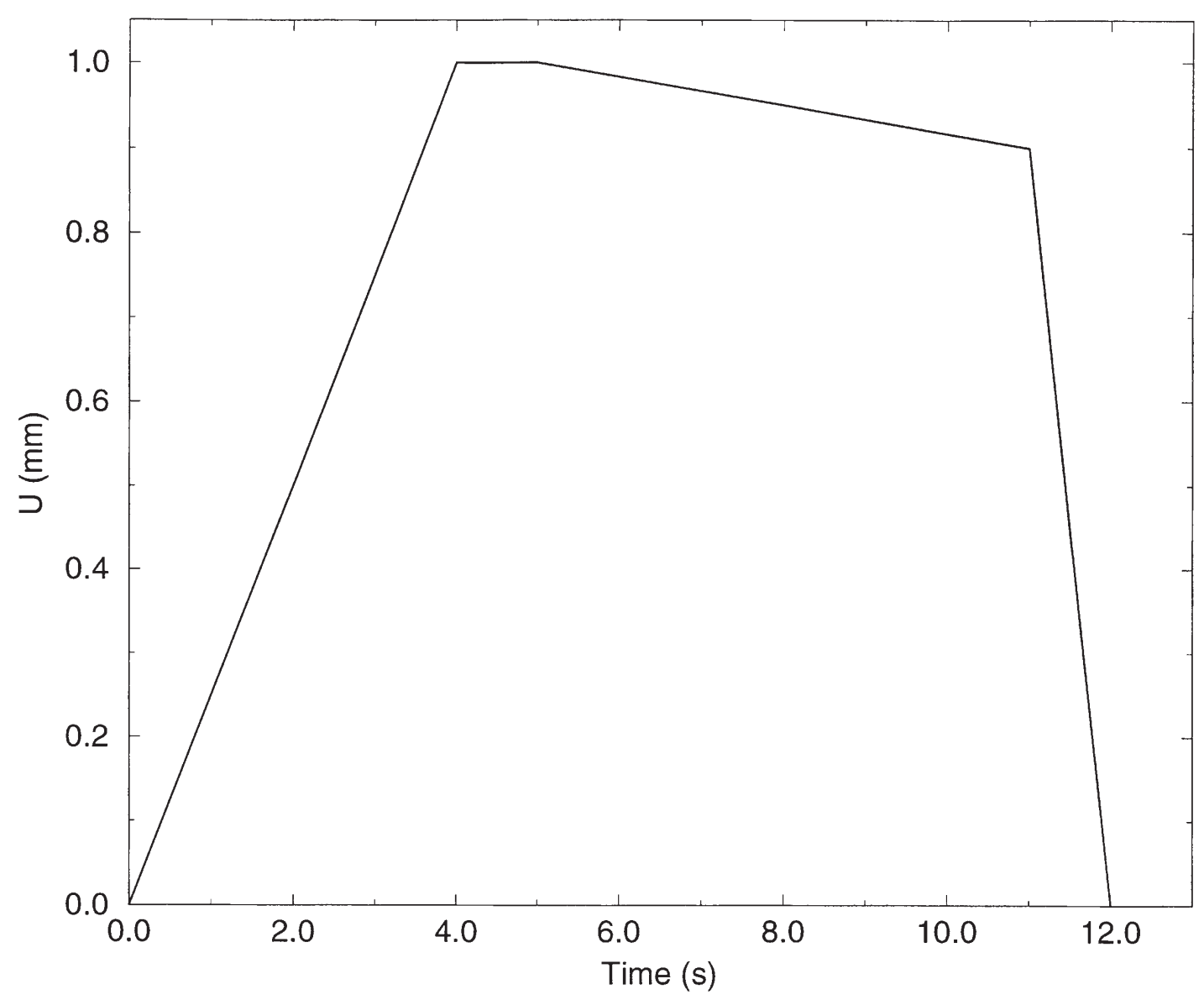

FIGURE 2 Loading history of the indentor.

The numerical gradient computation by the adjoint method has been compared with a computation by finite differences. The results for several points and directions showed less than $10 \%$ difference between the two methods.

The minimization algorithm was the quasi Newton BFGS algorithm with a line search obeying the Armijo selection rule [5].

The shape of the cost functional has been plotted in Figure 4 from a series of direct computations. We remark a smooth fiat valley which should not pose special difficulties to the identification.

\subsection{Identification Using Exact Measurements}

A first series of identifications have been performed with exact measurements. The results for different initial points are presented in Table I. The starting values for the algorithm have been at maximum 5 times smaller or 3 times larger than the real values. In all cases the final 


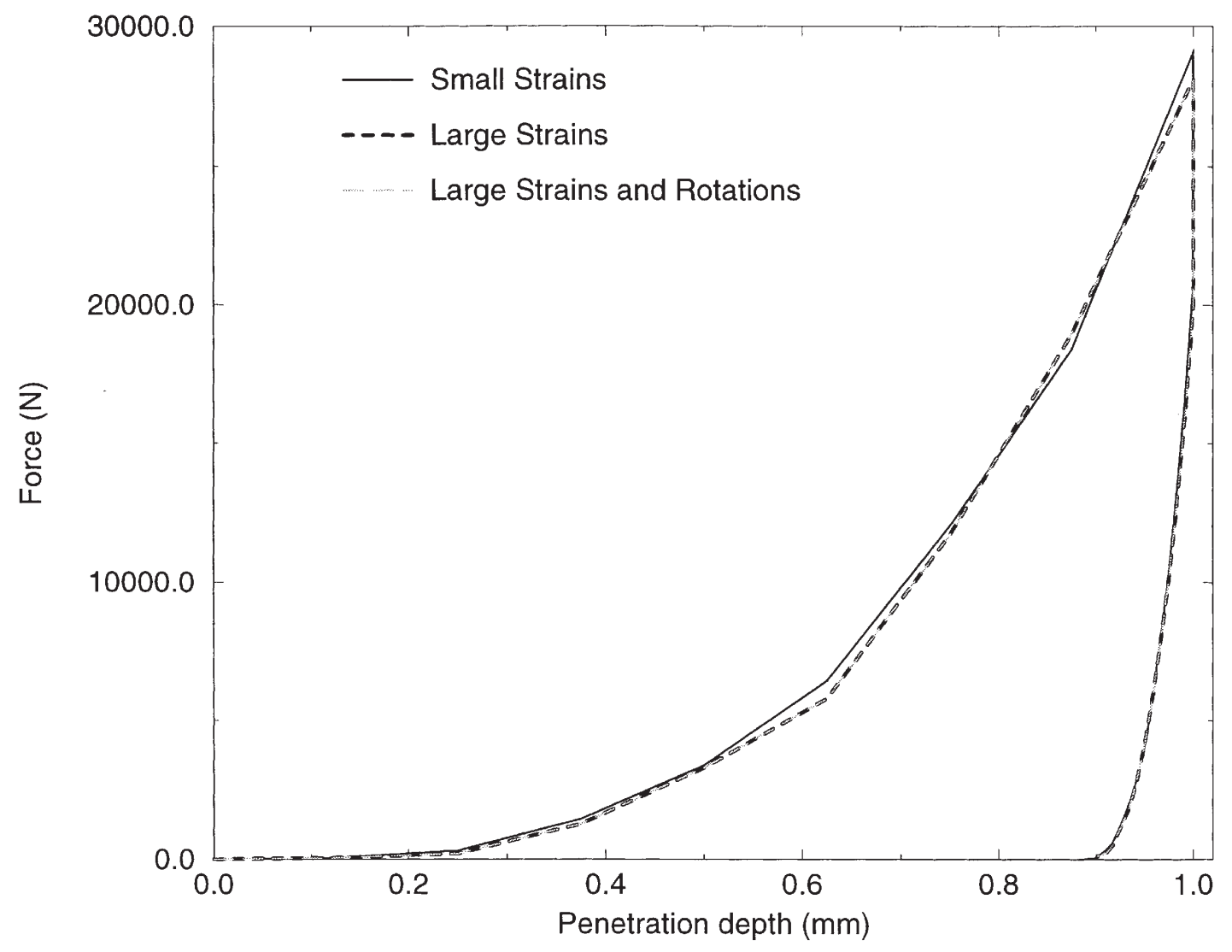

FIGURE 3 Indentation curve for small and large strains for the Maxwell material.

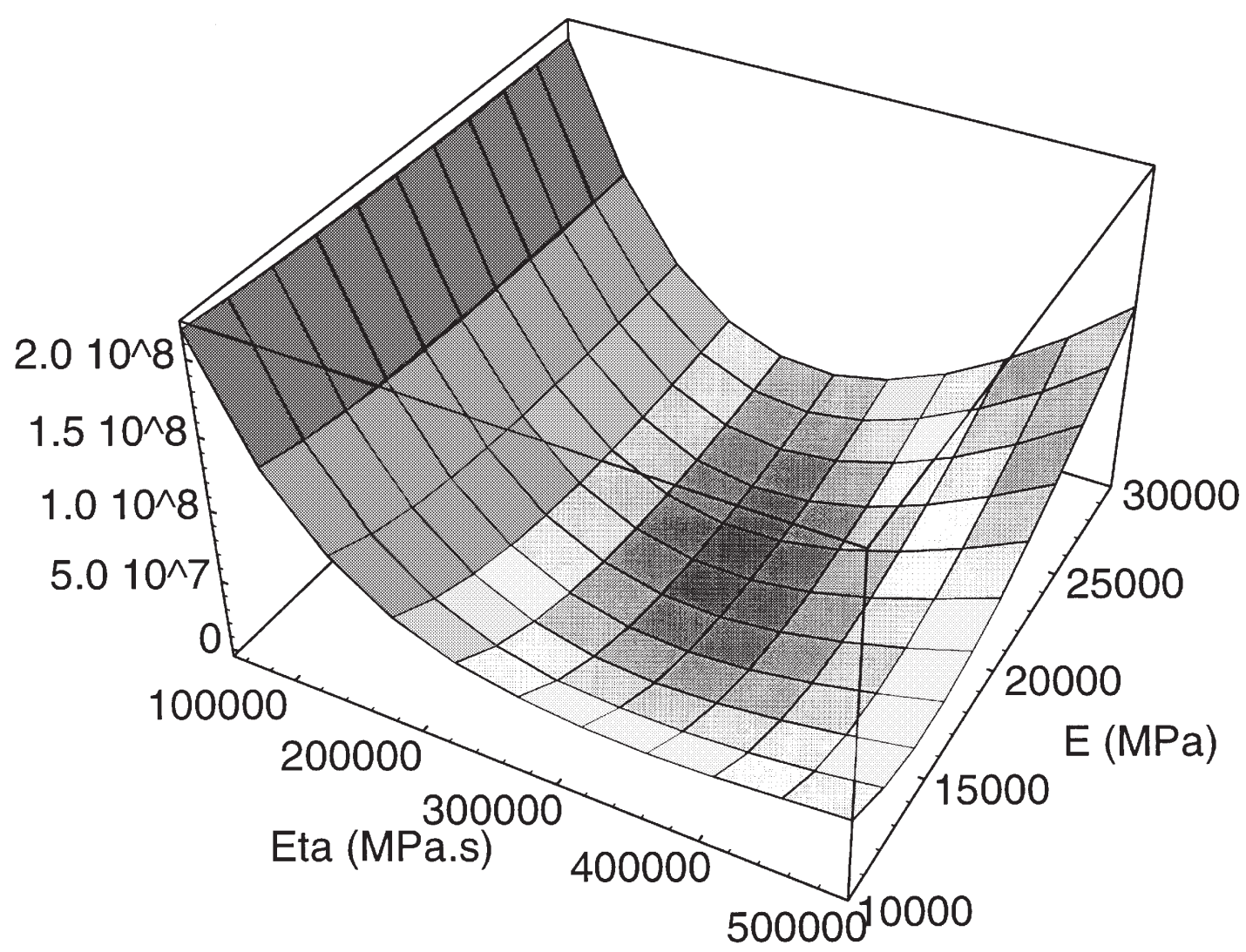

FIGURE 4 Shape of the cost functional $\mathcal{J}$ for the Maxwell material. 
TABLE I Identification results with exact measurements for the Maxwell material for the reference values $E^{\mathrm{ref}}=20000 \mathrm{MPa}, \eta^{\mathrm{ref}}=30000 \mathrm{MPa} \cdot \mathrm{s}$

\begin{tabular}{lc}
\hline$\left(E^{\text {ini }}, \eta^{\text {ini }}\right)$ & $\left(E^{\text {final }}, \eta^{\text {final }}\right)$ \\
$(M P a, M P a \cdot s)$ & $(M P a, M P a \cdot s)$ \\
\hline$(4000 ., 70000)$. & $(20002 ., 30004)$. \\
$(60000 ., 10000)$. & $(20002 ., 30009)$. \\
$(10000 ., 5000)$. & $(20002 ., 30006)$. \\
$(60000 ., 90000)$. & $(19999 ., 29999)$. \\
\hline
\end{tabular}

value was less than $0.02 \%$ from the value to be identified, after about 15 iterations. Some typical evolution path of the algorithm on the isovalues of the cost functional are plotted in Figure 5.

Figure 6 shows the real indentation curve in comparison to the

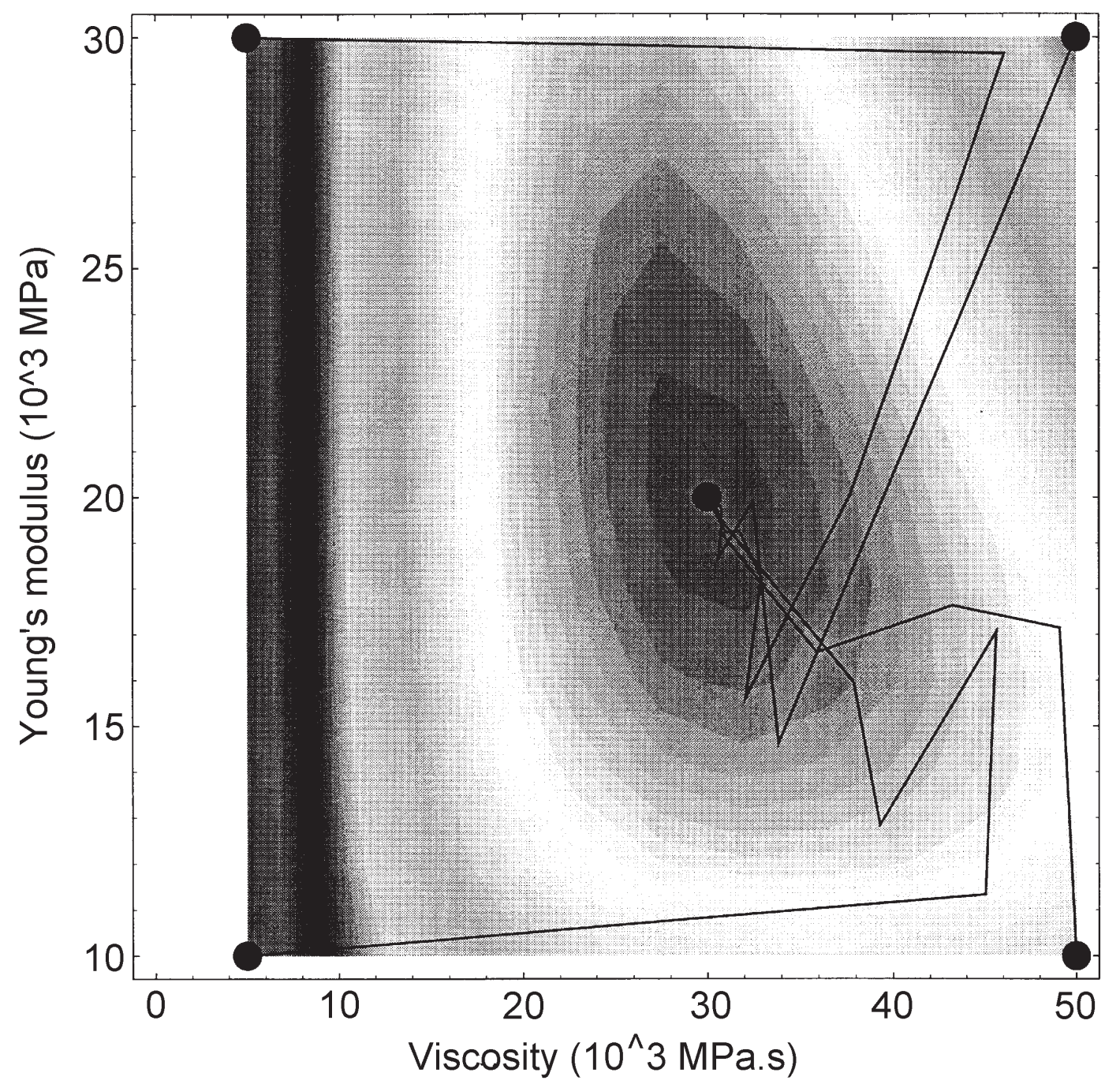

FIGURE 5 Path of the algorithm (exact measurements) for the Maxwell material. 


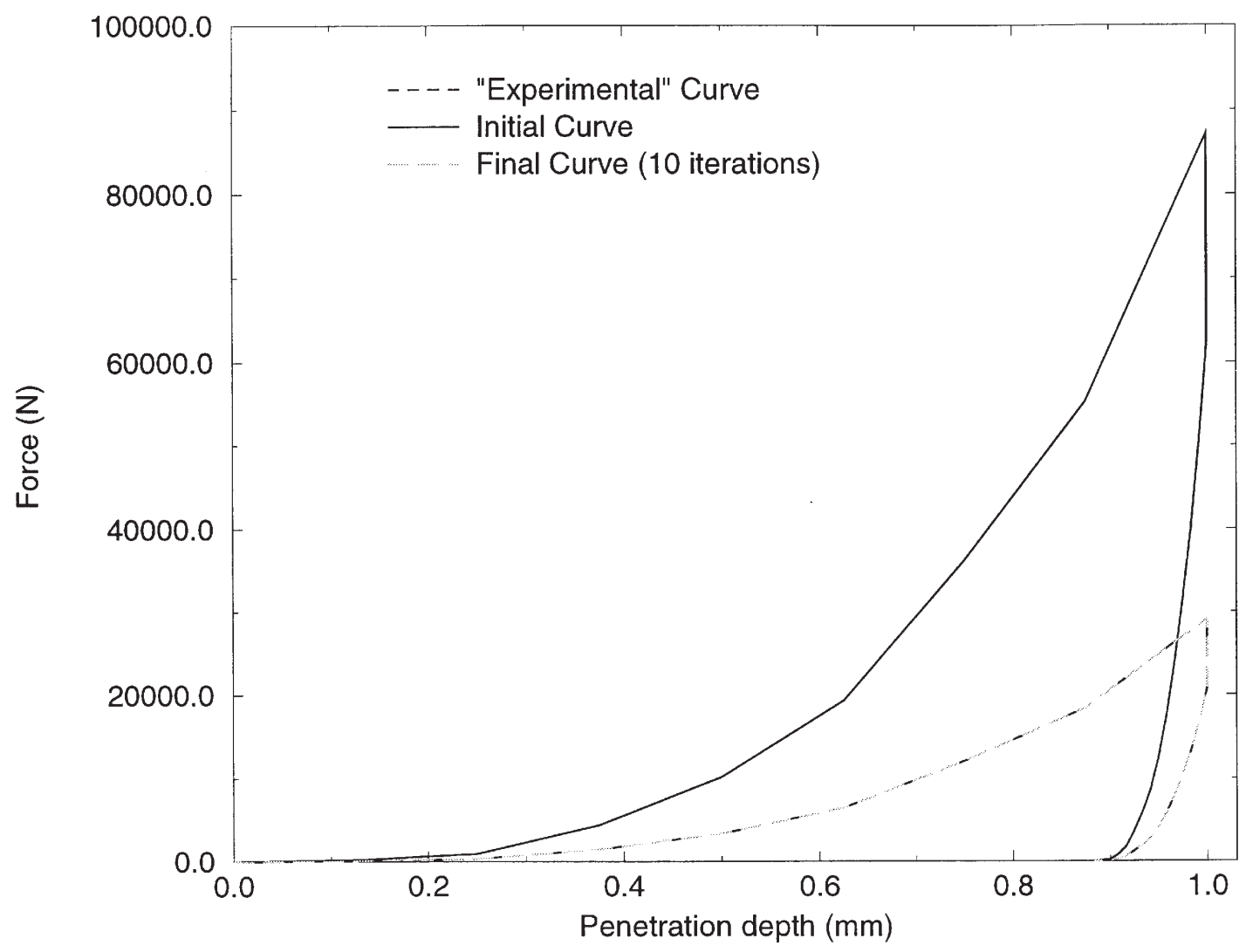

FIGURE 6 Evolution of the indentation curve with exact measurements.

initial and converged indentation curve. In terms of cost functional the algorithm brought its value from $\approx 10^{8}$ to $\approx 10^{1}$.

\subsection{Identification Using Measurements with Random Error}

In order to check the robustness of the identification procedure the simulated measurements have been perturbed by a $10 \%$ random noise. The results of several identifications using perturbed measurement data are presented in Table II. The first pair and the last pair of data are results coming from identification with different starting points but

TABLE II Identification results from measurements with random error for the Maxwell material

\begin{tabular}{lc}
\hline$\left(E^{\text {ini }}, \eta^{\text {ini }}\right)$ & $\left(E^{\text {final }}, \eta^{\text {final }}\right)$ \\
$(M P a, M P a \cdot s)$ & $(M P a, M P a \cdot s)$ \\
\hline$(4000 ., 70000)$. & $(20580 ., 30490)$. \\
$(10000 ., 5000)$. & $(20611 ., 30457)$. \\
$(60000 ., 10000)$. & $(19509 ., 29130)$. \\
$(60000 ., 90000)$. & $(19509 ., 29136)$. \\
\hline
\end{tabular}


with the same measurement perturbation. The identified values lie at $2.5 \%$ from the real values for both measurement perturbations.

The path of the algorithm on the isovalues of the perturbed cost functional is shown on Figure 7.

Figure 8 shows the real indentation curve $\left(E=210^{4} \mathrm{MPa}, \eta=3\right.$ $\left.10^{4} \mathrm{MPa} \cdot \mathrm{s}\right)$ in comparison to the initial $\left(E=610^{4} \mathrm{MPa}, \eta=910^{4} \mathrm{MPa}\right.$. s) and converged ( $E=1.9510^{4} \mathrm{MPa}, \eta=2.9110^{4} \mathrm{MPa} \cdot 10^{8}$ to $\approx 10^{4}$. This is minimal value of the cost functional where the algorithm could descent. However, there is a good agreement between the experimental and identified indentation curve.

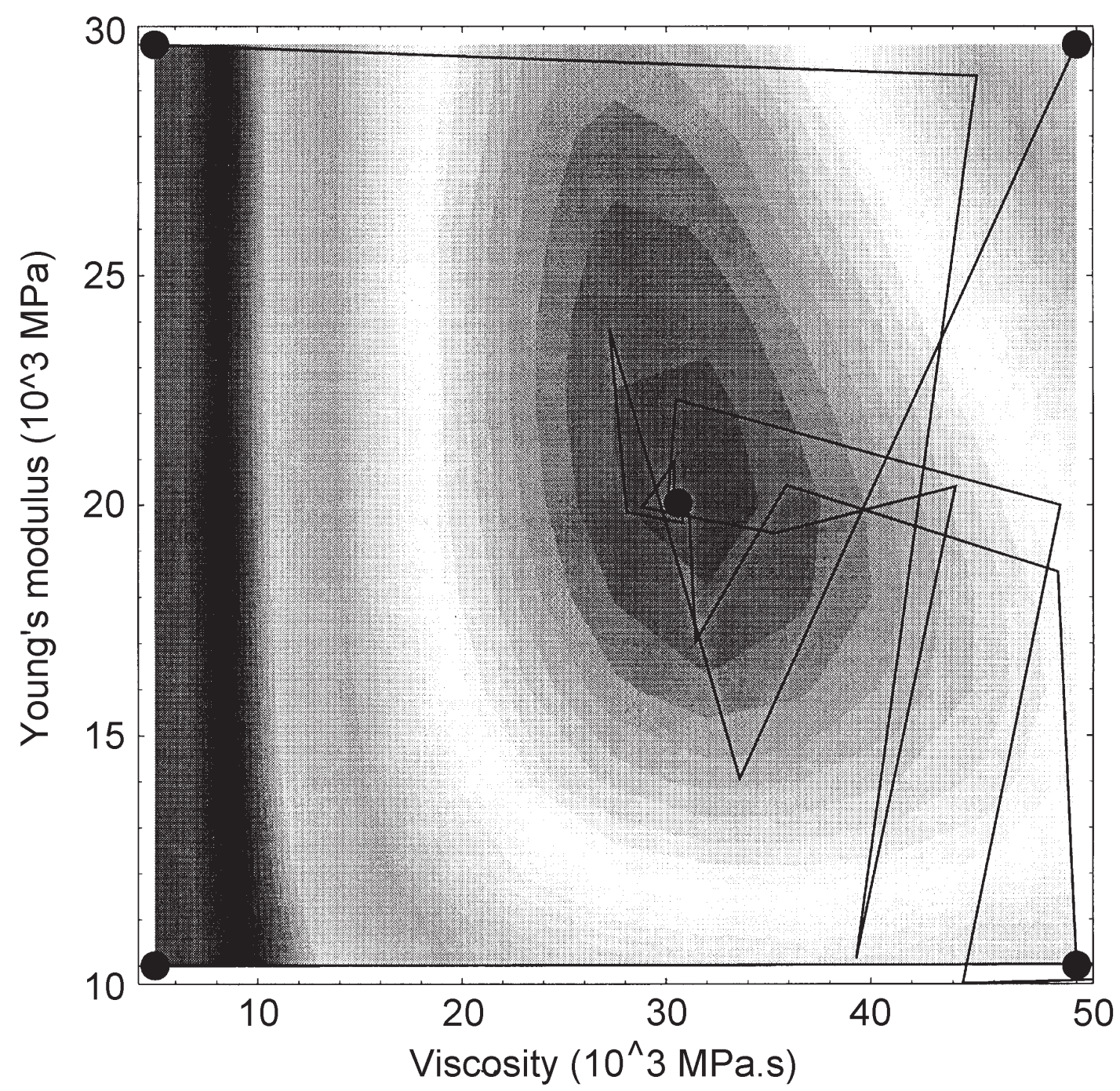

FIGURE 7 Path of the algorithm (measurements with random error) for the Maxwell material. 


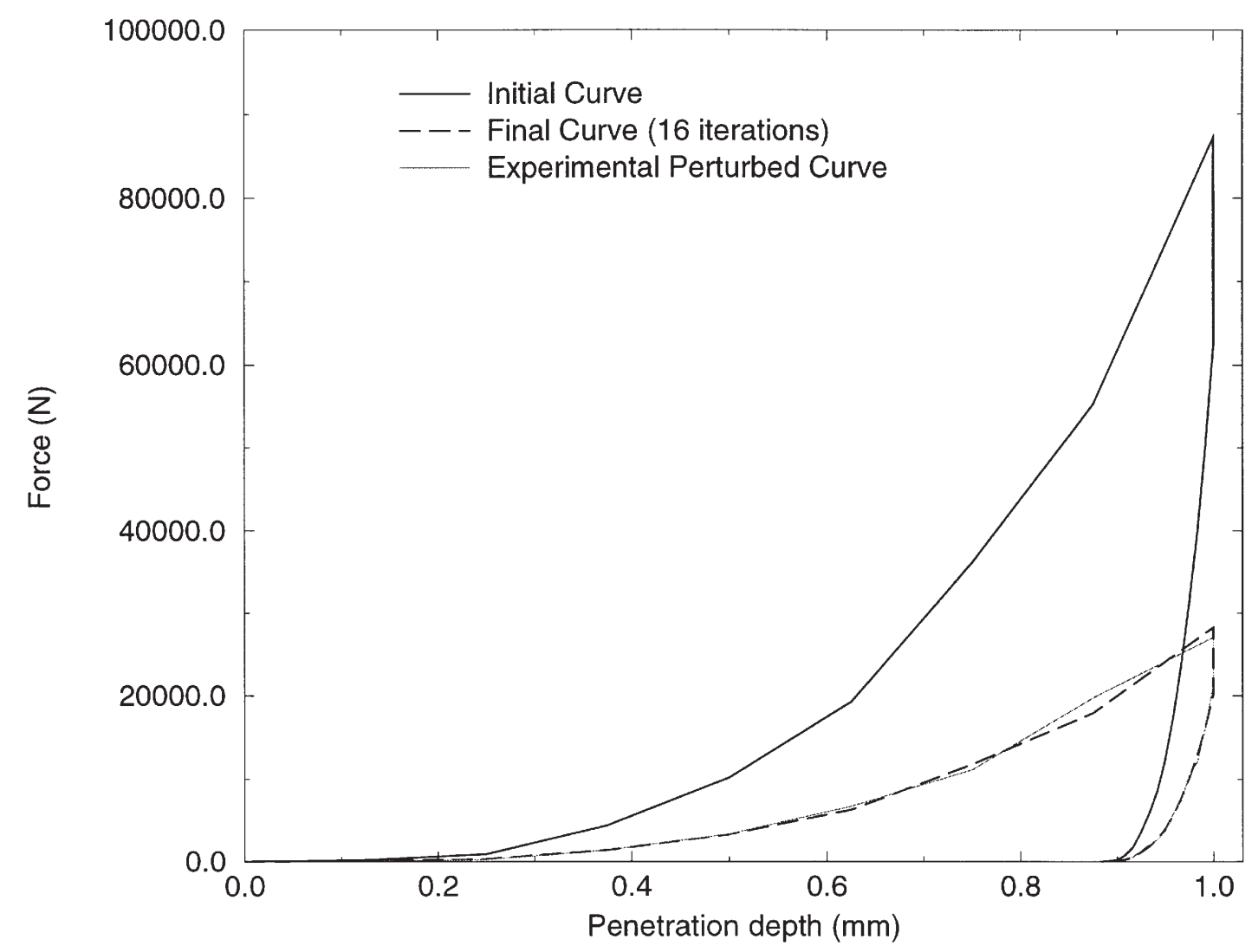

FIGURE 8 Evolution of the indentation curve from measurements with random error for the Maxwell material.

\section{NUMERICAL EXAMPLE FOR THE NORTON-HOFF MATERIAL}

We recall that the viscoplastic Norton-Hoff constitutive law is expressed as:

$$
\begin{gathered}
\varepsilon\left(\Delta \mathbf{u}_{i}\right)=\mathbf{S}: \Delta \boldsymbol{\sigma}_{i}+\Delta \varepsilon_{i}^{p} \quad \text { where } \Delta \varepsilon_{i}^{p}=\mathbf{M}: \boldsymbol{\sigma}_{i} \Delta t \\
S_{i j k l}=\frac{1}{E}\left((1+\nu) \delta_{i k} \delta_{j l}-\nu \delta_{k l} \delta_{i j}\right) \\
\Delta \varepsilon_{i}^{p}=\frac{3}{2}\left\langle\frac{\left(\sigma_{i}\right)_{\mathrm{eq}}-\sigma^{Y}}{K}\right\rangle^{m} \frac{\tilde{\boldsymbol{\sigma}}_{i}}{\left(\sigma_{i}\right)_{\mathrm{eq}}} \Delta t
\end{gathered}
$$

As explained before, this constitutive law enters the formalism of the standard generalized materials without work-hardening. In comparaison with the Maxwell viscoelasticity two difficulties have been adjoined a yield limit and a nonlinearity represented by the power law. 


\subsection{Direct Calculations}

The direct computations have been programmed in the same framework as previously presented and similar meshes and loading histories (Fig. 2) have been used. The integration of the direct constitutive law was performed using a build in forward Euler scheme (implicit) under the hypothesis of small strains.

\subsection{Identification Procedure}

The identification procedure is based on minimization of the cost functional $\mathcal{J}$ (16) using the same algorithm as before.

The "experimental" curve was simulated by finite element calculations with $E=10^{5} \mathrm{MPa}, \quad \nu=0.3, \quad K=1500 \mathrm{MPa} \cdot \mathrm{s}^{1 / m}, \quad m=5$, $\sigma^{Y}=500 \mathrm{MPa}$.

The gradient has been computed using the adjoint state method with the expression (40) after solving the direct problem $(\mathcal{P})$ and the adjoint problem $\left(\mathcal{P}^{\star}\right)$.

Opposite to the Maxwell case, the adjoint constitutive law is different from the direct one and can be expressed as:

$$
\varepsilon\left(\Delta \mathbf{u}_{i}^{*}\right)=\mathbf{S}: \Delta \boldsymbol{\sigma}_{i}^{*}+\mathbf{M}: \boldsymbol{\sigma}_{i}^{*}
$$

where:

$$
\begin{aligned}
\mathbf{M} & =-\frac{3}{2}\left\langle\frac{\left(\sigma_{i}\right)_{\mathrm{eq}}-\sigma^{Y}}{K}\right\rangle^{m} \frac{1}{\left(\boldsymbol{\sigma}_{i}\right)_{\mathrm{eq}}} \mathbf{J} \\
& +\frac{\tilde{\boldsymbol{\sigma}}_{i} \otimes \tilde{\boldsymbol{\sigma}}_{i} 9}{\left(\boldsymbol{\sigma}_{i}\right)_{\mathrm{eq}}^{2}} \frac{9}{4}\left[\left\langle\frac{\left(\sigma_{i}\right)_{\mathrm{eq}}-\sigma^{Y}}{K}\right\rangle^{m} \frac{1}{\left(\boldsymbol{\sigma}_{i}\right)_{\mathrm{eq}}^{2}} \frac{m}{K}\left\langle\frac{\left(\sigma_{i}\right)_{\mathrm{eq}}-\sigma^{Y}}{K}\right\rangle^{m-1}\right. \\
J_{i j k l} & =\left(\delta_{i k} \delta_{j l}-\frac{1}{3} \delta_{k l} \delta_{i j}\right)
\end{aligned}
$$

This formula already expresses a forward Euler integration scheme which has been programmed as such in the code.

One can remark that the adjoint constitutive law is a viscoelastic, nevertheless anisotropic and nonhomogenous. 


\subsection{Identification Using Exact Measurements}

A series of identifications have been performed with exact measurements on two meshes with different coarsness (tests $1-5$ and 6-8) and the results for different initial points are presented in Table III.

The starting values for the identification are in this case just $\approx 30 \%$ from the real values. This choice has been motivated by the slow convergence of the direct computation for the Norton-Hoff material.

One can remark that the parameters have been identified within $\approx 5 \%$ of the real values for tests $1,2,7$ and 8 . In the other cases, only the Young's modulus $E$ and the viscosity $K$ have been reasonably identifiyed ( $1 \%$ error for $E$ and $1-10 \%$ for $K$ ). The power coefficient $m$ and the yield limit $\sigma^{Y}$ are in these cases still far away from the real values. However the final computed indentation curves are always superposed with the experimental curve, meaning that the minimization of the geometrical distance between them has been achived.

The initial and final parameters in tests 5 present a peculiar property. As can be seen in Figure 9, both parameters sets produce the same loading curve as the experimental one. The best identified parameter in this case, was the Young's modulus. This result is a new confirmation of the importance of the unloading part of the experimental curve in the identification of the Young's modulus as already observed in a series of previous papers [16,17].

The previous results are a direct consequence of the poor sensitivity of the cost functional in this region of the parameter space. In order to illustrate this property we have plotted the values of the cost

TABLE III Identification results with exact measurements for the Norton-Hoff material for the reference values $E^{\text {ref }}=100000 . \mathrm{MPa}, K^{\mathrm{ref}}=1500 . \mathrm{MPa} . \mathrm{s}^{1 / 5} \mathrm{~m}^{\mathrm{ref}}=5$. and $\sigma^{Y \text { ref }}=500 . \mathrm{MPa}$

\begin{tabular}{lcccrrrrr}
\hline Test & $\begin{array}{c}E^{\text {ini }} \\
M P a\end{array}$ & $\begin{array}{c}K^{\text {ini }} \\
(M P a \cdot s)^{1 / m}\end{array}$ & $m^{\text {ini }}$ & $\begin{array}{r}\sigma^{Y \text { ini }} \\
M P a\end{array}$ & $\begin{array}{r}E^{\text {final }} \\
M P a\end{array}$ & $\begin{array}{c}K^{\text {final }} \\
(M P a \cdot s)^{1 / m}\end{array}$ & $m^{\text {final }}$ & $\begin{array}{c}\sigma^{Y \text { final }} \\
M P a\end{array}$ \\
\hline 1 & 130000. & 800. & 2. & 1000. & 99664.3 & 1533.36 & 5.38417 & 446.414 \\
2 & 150000. & 1000. & 3. & 1000. & 100228.0 & 1524.64 & 5.00296 & 491.795 \\
3 & 150000. & 2000. & 3. & 200. & 99429.3 & 1598.24 & 6.18529 & 343.533 \\
4 & 150000. & 2000. & 8. & 1000. & 99288.9 & 1657.20 & 6.98908 & 246.959 \\
5 & 70000. & 2000. & 8. & 200. & 99126.5 & 1682.47 & 6.94556 & 238.366 \\
6 & 70000. & 3000. & 8. & 250. & 100598.0 & 1451.36 & 4.24316 & 602.651 \\
7 & 70000. & 1000. & 3. & 250. & 99630.1 & 1521.18 & 4.94161 & 497.229 \\
8 & 150000. & 1000. & 3. & 250. & 99778.0 & 1516.32 & 5.28676 & 464.255 \\
\hline
\end{tabular}



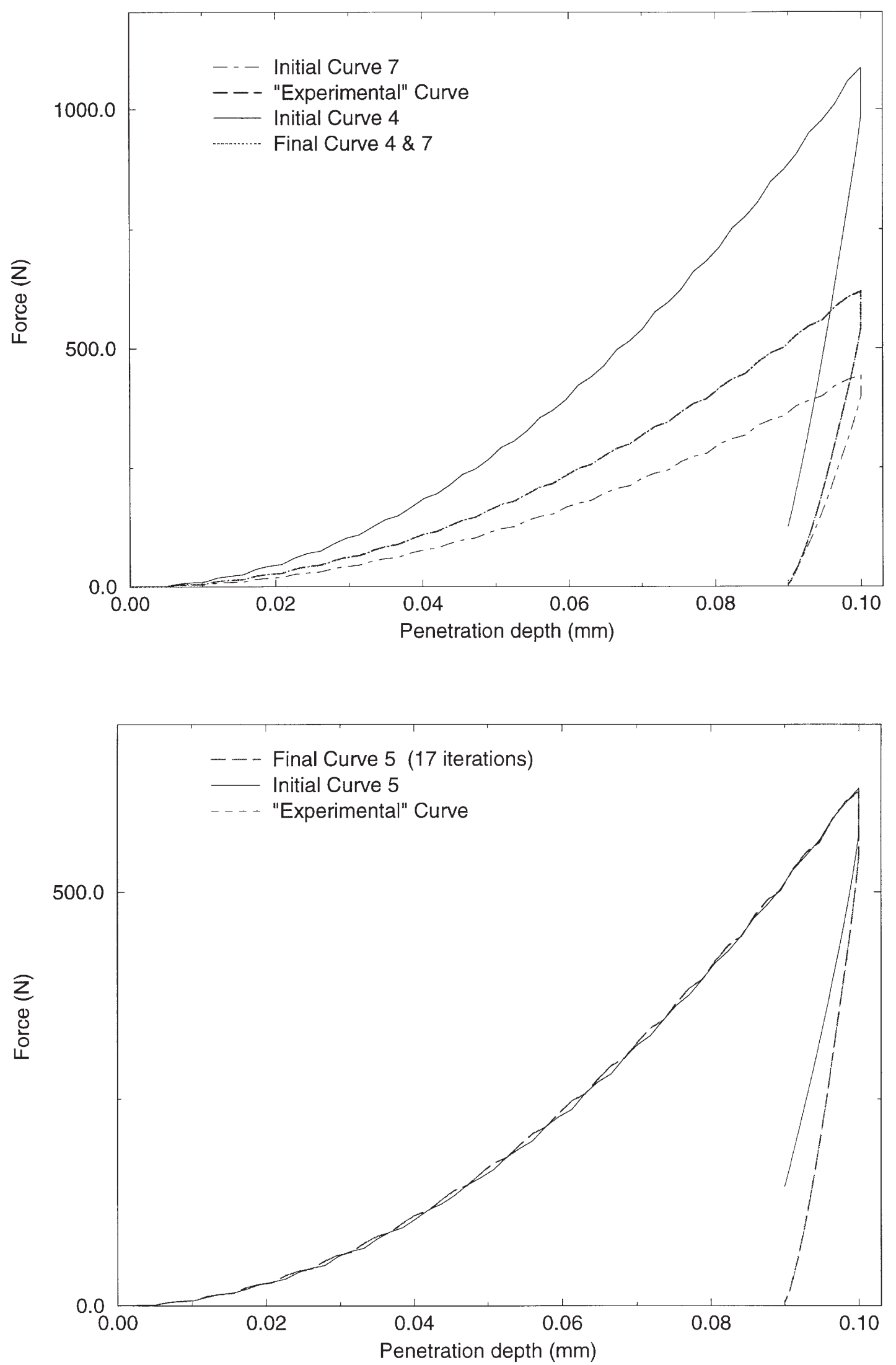

FIGURE 9 Evolution of the indentation curve with exact measurements for the Norton-Hoff material. 


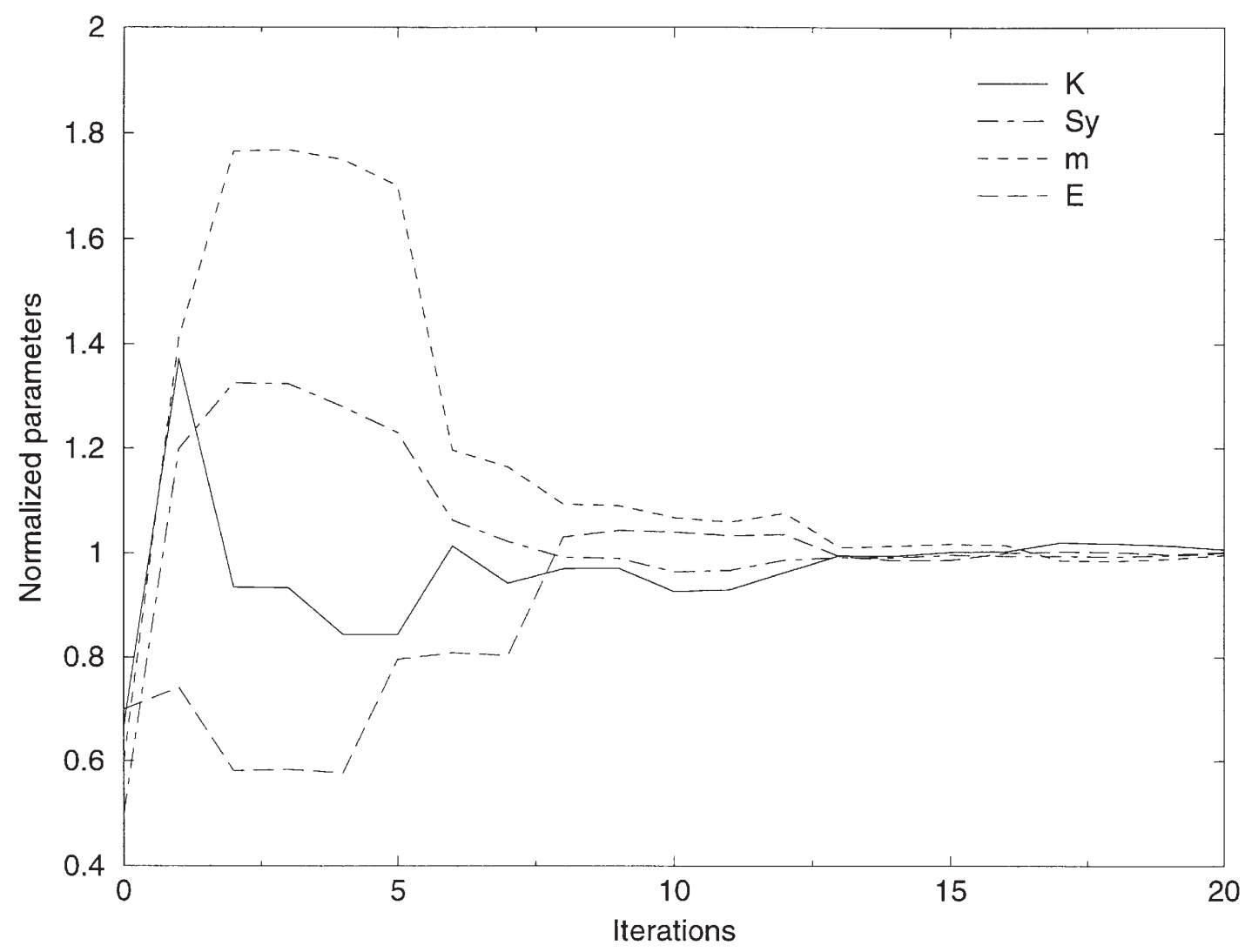

FIGURE 10 Convergence of the normalized parameters for the Norton-Hoff material (test 7).

functional $\mathcal{J}$ on a plane of the parameter space determined by the following points:

- the reference point: $\mathbf{c}=(100000,1500,5,500)$

- the initial point in test 5: $\mathbf{c}=(70000,2000,8,200)$

- the final point in test 5: $\mathbf{c}=(99126,1682,6.95,238)$

Using a coordinate parametrization given by two orthogonal vectors, the parameter sets in this plane can be generated using the following formula:

$$
\begin{aligned}
E & =100000-1315.4 a-80362.7 b \\
K & =1500+273.917 a+714.075 b \\
m & =5+2.93482 a+1.08013 b \\
\sigma^{y} & =500-394.32 a+142.208 b
\end{aligned}
$$

where $a, b$ are two real parameters.

In Figure 11 we represented the values of the cost functional for the parameter sets generated with $(a, b) \in[-1,1] \times[-0.5,0.5]$. Two 


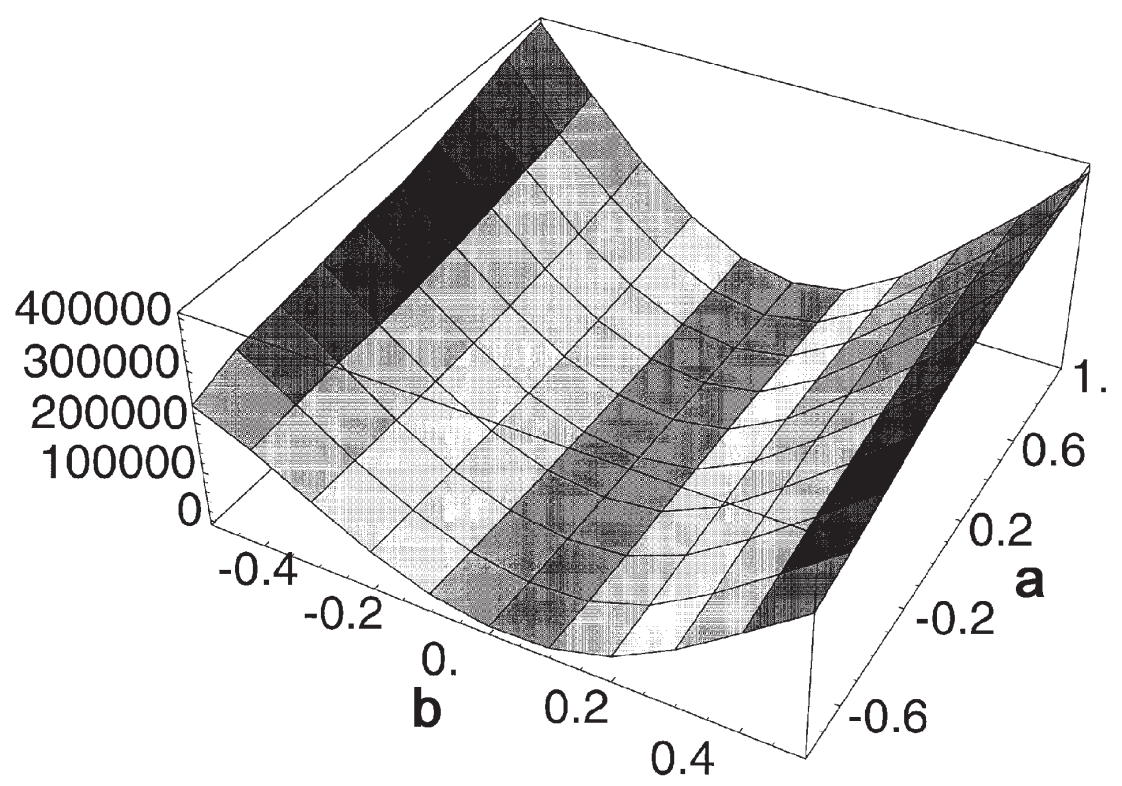

FIGURE 11 The values of the cost functional $\mathcal{J}$ on the "critical" plane (see Eq. (??)) of the parameter space.

extreme values $(141200,923,2.11,744)$, i.e., $(a, b)=(-1,-0.5)$ and $(58500,2131,8.47,176)$, i.e., $(a, b)=(1,0.5)$ show how spanned are the values covered in this region. The cost functional presents a deep and long valley where it is very difficult to converge to the minimum. A close inspection of the obtained values show however that there is a unique minimum in this region.

\section{CONCLUSION}

The identification of the parameters of a standard generalized non hardening constitutive law from indentation tests was presented. It has been shown that the adjoint state method can be extended to contact problems using Lagrange multipliers.

The efficiency of the method has been studied on numerical examples for an indentation problem in the case of Maxwell viscoelatic and Norton-Hoff viscoplastic constitutive laws.

Even if no precise proof of uniqueness and stability has been provided, one can remark that for the Maxwell material the cost functional is almost convex and presents a unique minimum (see Fig. 4). For other values of the material parameters $\mathbf{c}=(E, \eta)$ we have obtained similar shapes of the cost functional. It is important to state 
that for the Maxwell material identifications started with completely different values did converge to the same parameter set. All these remarks suggest that this inverse identification problem has a unique solution.

In the case of the Norton-Hoff material the numerical examples presented a lack of sensitivity of the cost functional with regards to certain parameters. However the Young moduli and the viscosity are well identified. In spite of the lack of sensitivity it seems that the identification problem has a unique minimum.

In order to improve the presented solution, different possibilities are available: to increase the number of experimental measures for example by varying the indentation rate, to change the expression of the cost functional like the error on constitutive law or crossed differences $\left(\mathcal{J}=\left(U^{\exp }-U^{\text {comp }}\right) \times\left(F^{\text {comp }}-F^{\exp }\right)\right.$ see [20]). These questions do not depreciate the presented approach as the technique for the gradient computations remains valid. They rather demand a study of the strategy of identification and of the identifiability of a certain family of constitutive laws.

\section{Acknowledgments}

The authors would like to thank Said Taheri, Stéphane Andrieux and Laurent Bourgeois for helpful discussions and acknowledge the financial support of EDF-Electricité de France for this work.

\section{References}

[1] Bourgeois, L. (1998). Contrôle optimal et problèmes inverses en plasticité, Ph.D. thesis of the École Polytechnique.

[2] Bui, H. D. (1994). Inverse problems in the mechanics of materials: an introduction, CRC Press, Boca Raton.

[3] Castem2000 Finite Element Code http : //www.castem.org.

[4] Constantinescu, A. and Tardieu, N. (1998). The adjoint state method applied to material identification through indentation tests, In: Advances in Finite Element Procedures and Techniques, Civil Comp Press.

[5] Gill, P. E., Murray, W. and Wright, M. H. (1981). Practical optimization, Academic Press.

[6] Halphen, B. and Nguyen, Q. S. (1975). Sur les matériaux standards généralisés, Journal de Mécanique.

[7] Hasanov, A. and Seyidmamedov, Z. (1995). The solution of an axisymmetric inverse elastoplastic problem using penetration diagrams, Int. J. Non-Linear Mech., Vol. 30. 
[8] Hasanov, A. (1995). An inverse problem for an elastoplastic medium, SIAM J. Appl. Math., 55(5), 1736-1752.

[9] Hasanov, A. (1998). Inverse coefficient problems for elliptic variational inequalities with a nonlinear monotone operator, Inverse Problems, 14(5), 1151-1170.

[10] Hilding, D. Klarbring, A. and Petersson, J. (1999). Optimization of structures in unilateral contact, ASME Applied Mechanics Review, 52(4), 139-157.

[11] Jayaraman, S., Hahn, G. T., Oliver, W. C., Rubin, C. A. and Bastias, P. C. (1998). Determination of monotonic stress-strain curve of hard metals from ultra-low-load indentation tests, Int. J. Solids Structures, 35(5-6).

[12] Johnson, K. L. (1985). Contact mechanics, Cambridge University Press.

[13] Kikuchi, N. and Oden, J. T., Contact problems in elasticity: a study of variational inequalities and finite element methods, SIAM, Philadelphia, 1988.

[14] Koguchi, H. (1999). Determination of mechanical properties of thin films and functional gradient materials using an inverse technique, Conference on Inverse Problems in Engineering: Theory and Practice, ASME.

[15] Lions, J. L., Contrôle optimal des systèmes gouvernés par des équations aux dérivées partielles, Dunod, 1968.

[16] Loubet, J. L., Georges, J. M., Marchesini, O. and Meille, G. (1984). Vickers indentation curve for a magnesium oxide $(\mathrm{MgO}), J$. of Tribology, 106.

[17] Oliver, W. C. and Pharr, G. M. (1992). An improved technique for determining hardness and elastic moduli used load and displacement sensing indentation experiments, J. Mater. Res., 7(6).

[18] Tabor, D. (1951). Hardness of metals, Oxford University Press.

[19] Taljat, B., Zacharia, T. and Kosel, F. (1998). New analytical procedure to determine stress-strain curve from spherical indentation data, Int. J. Solids Structures, 35(33).

[20] Tardieu, N. and Constantinescu, A., On the determination of elastic coefficients from indentation tests, accepted for publication in Inverse Problems. 